\title{
ON THE CORADICAL OF A FINITE-DIMENSIONAL HOPF ALGEBRA
}

\author{
DAVID E. RADFORD ${ }^{1}$
}

\begin{abstract}
Examples are constructed showing that the coradical of a finite-dimensional Hopf algebra over any algebraically closed field is not necessarily a subalgebra (hence the Jacobson radical is not a Hopf ideal in general). The square of the antipode may induce a permutation on the simple subcoalgebras of dimension $>1$ of arbitrarily high order.
\end{abstract}

0 . Introduction. In this paper we discuss the coradical (and thus naturally the Jacobson radical) of a finite-dimensional Hopf algebra over a field $k$. Over any algebraically closed field finite-dimensional examples are constructed such that the coradical is not a sub-Hopf algebra (hence the Jacobson radical is not necessarily a Hopf ideal). In [2] it is shown that the square of the antipode maps each simple subcoalgebra into itself in the cosemisimple case. In general this does not happen; there exist finite-dimensional Hopf algebras over any algebraically closed field $k$ with antipode $s$ of order $2 n$ ( $n$ large enough) such that $t=s^{2}$ induces a permutation on the simple subcoalgebras of dimension $>1$ or order $n$.

The Hopf algebras in this paper arise surprisingly enough as dual Hopf algebras of pointed ones which have a relatively simple description. The basic ideas of the constructions are taken from [5] and [3]. The moderate amount of notation and results concerning Hopf algebras we assume can be found in [4], and the algebra we take for granted is adequately covered in [1]. All vector spaces, coalgebras, Hopf algebras, etc. will be over a field $k$.

1. Preliminaries. The examples we construct in this paper will be dual Hopf algebras of finite-dimensional pointed Hopf algebras. The basic building block is Taft's example of [5] as described below.

1.1. Let $k$ be a field containing a primitive $n$th root of unity $\omega, n>1$. The free algebra $\mathcal{H}=k\{A, Z\}$ on noncommuting indeterminants $A$ and $Z$ is a bialgebra where $\Delta: \mathcal{H} \rightarrow \mathcal{H} \otimes \mathcal{H}$ is determined by

$$
\Delta A=A \otimes A \text { and } \Delta Z=Z \otimes A+1 \otimes Z,
$$

Received by the editors September 3, 1974. 16A64.

AMS (MOS) subject classifications (1970). Primary 16A24; Secondary 16A21,

Key words and phrases. Hopf algebra, Jacobson radical, order of antipode on coradical.

1 Research done while the author was a visitor to the Institute for Advanced Study, Princeton, N. J., and supported by NSF Grant GP- 42922. 
and $\epsilon: \mathcal{H} \rightarrow k$ is determined by

$$
\epsilon(A)=1 \quad \text { and } \quad \epsilon(Z)=0 .
$$

The ideal $\gamma$ of $\mathcal{H}$ generated by $A^{n}-1, Z A-\omega A Z$, and $Z^{n}$ is a bi= ideal, and $T_{n}=\mathcal{H} / \gamma$ is a Hopf algebra with antipode of order $2 n . T_{n}$ has for a basis (denoting cosets by small case letters) the set $\left\{a^{i} z^{j}: 0 \leq i, j<n\right\}$. Thus $\operatorname{dim} T_{n}=n^{2}$.

We will maintain the convention of denoting cosets by small case letters in $\delta, \delta 2,3$.

Taft's example $T_{n}$ and its dual $T_{n}^{*}$ are isomorphic as Hopf algebras. For let $a \in \operatorname{Alg}\left(T_{n}, k\right)=G\left(T_{n}^{*}\right)$ and $\mathscr{E} \in T_{n}^{*}$ be determined by

$$
\alpha(h)=\left\{\begin{array}{l}
\omega: h=a, \\
0: h=z,
\end{array} \quad \text { and } \quad Z(h)=\left\{\begin{array}{l}
1: h=a^{l} z, \\
0: h=a^{l} z^{p}, p \neq 1 .
\end{array}\right.\right.
$$

One can check that the map $T_{n} \rightarrow T_{n}^{*}$ determined by $a \mapsto a$ and $z \mapsto \stackrel{g}{二}$ is an isomorphism of Hopf algebras.

The following observations will be useful in 1.3 and $\$ 3$.

1.2. Let $A$ be an algebra over $k$, and $a, b \in A$ satisfy $a b=\omega b a$ for some $0 \neq \omega \in k$. Then:

(a) $(a b)^{n}=\omega^{-(n-1) n / 2} a^{n} b^{n}$ for $n \geq 1$.

(b) If $\omega$ is a primitive $n$th root of unity then $(a+b)^{n}=a^{n}+b^{n}$.

(a) follows easily by induction, and (b) is essentially proven in [5].

In order to analyse the structure of the Hopf algebras in $\$ \$ 2$ and 3 it will be necessary to show that certain algebras are semisimple. The next proposition provides sufficient criteria for our purposes.

1.3. Proposition. Let $B$ be an algebra over a field $k$.

(a) Suppose $B$ is generated by $a$ and $z$ which satisfy $a^{n}=z^{n}=1$ and $z a=\omega a z$, where $\omega \in k$ is a primitive nth root of unity, $n>1$. Then $B \simeq$ $M(n, k)$.

(b) Suppose $k$ is algebraically closed, and $B$ is generated by $a, x, y$ which satisfy $a^{n}=x^{n}=y^{n}=1, x a=\omega a x, y a=\eta a y$, and $x y=\eta^{-1} y x$, where $\omega, \eta \in k$ are primitive nth roots of unity. Then there is $a \xi \in k$ and $q>0$ such that $\left(\xi a^{n-q} x^{q}\right)^{n}=1$, and any maximal ideal is of the form $\mathbb{M}_{l}=$ $\left(y-\xi \omega^{l} a^{n-q} x^{q}\right)$ for some $0 \leq l<n$. Furthermore $B \simeq M(n, k) \times \cdots \times M(n, k)$ (p-times) where $p \leq n$.

Proof. (a) Let $V$ be a vector space over $k$ with basis $v_{0}, \ldots, v_{n-1}$, and define $\mathbb{G}, \mathcal{E} \in \operatorname{End}(V)$ by $\mathbb{Q}\left(v_{i}\right)=v_{i+1}$ (adding subscripts modulo $n$ ) and $\mathcal{Z}\left(v_{i}\right)=\omega^{i-1} v_{i}$. Then $\operatorname{End}(V)$ with $\mathbb{Q}$ and $\mathcal{Z}$ satisfies the hypothesis of 1.3(a).

Let $\{t=k\{A, Z\}$ be the free algebra on noncommuting indeterminants $A$ 
and $Z$, and let End $(V) \leftarrow \mathfrak{U} \rightarrow B$ denote the obvious surjections. Let $\gamma$ be the ideal of $\mathcal{U}$ generated by $A^{n}-1, Z^{n}-1$, and $Z A-\omega A Z$. Then $\operatorname{dim} \mathfrak{U} / \gamma$ $\leq n^{2}$, so we have factorizations $\operatorname{End}(V) \simeq \mathfrak{d} / \gamma \rightarrow B$. This proves (a).

(b) $\eta=\omega^{q}$ for some $0<q<n$. Set $\mathcal{Y}=a^{n-q} x^{q}$. Then one can check

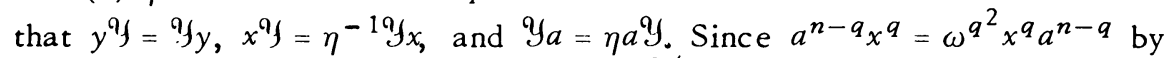
$1.2(\mathrm{a})$, we compute that $\mathfrak{Y}^{n}=\omega^{-(n-1) n q^{2 / 2}} 1$. Fix $\xi \in k$ satisfying $\xi^{n}=$ $\omega^{(n-1) n q^{2 / 2}}$, and consider the ideal $\mathbb{M}_{l}=\left(y-\xi_{\omega} l^{l} n-q_{x}^{q}\right)$. We will show that $\mathbb{M}_{l}$ is maximal (or $B$ ), and if maximal then $B / \mathbb{M}_{l} \simeq M(n, k)$.

It is clear that $M_{l}$ is generated as a left ideal by $y-\xi \omega^{l} a^{n-q} x^{q}$, and that $\mathbb{M}_{l}+\mathbb{M}_{l^{\prime}}=B$ for $0 \leq l, l^{\prime} \leq n$ distinct. Thus $\bigcap_{l=0}^{n-1} \mathbb{M}_{l}=\mathbb{M}_{0} \ldots . \mathbb{M}_{n-1}$. But the latter is generated by

$\left(y-\xi a^{n-q} x^{q}\right)\left(y-\omega \xi a^{n-q} x^{q}\right) \ldots\left(y-\omega^{n-1} \xi a^{n-q} x^{q}\right)=y^{n}-\left(\xi a^{n-q} x^{q}\right)^{n}=0$.

Thus we can conclude that $B \simeq B / M_{0} \times \cdots \times B / M_{n-1}$. If $M_{l}$ is proper, then part (a) implies that $B / M_{l} \simeq M(n, k)$. This completes the proof of (b). Q.E.D.

Remark. In 1.3 if $a^{n}=1$, etc. is replaced by $a^{n}=\alpha 1$, whe re $0 \neq \alpha \in k$, we can conclude that $B$ is semisimple. For if $\bar{k}$ is an algebraic closure of $k$, then 1.3 guarantees that $B \otimes \bar{k}$ is semisimple.

2. The order of $s$ on the coradical. The examples in this section will show that the order of the antipode $s$ on the coradical can be $2 n$ for $n \geq 1$. In every case the Hopf algebra is generated as an algebra by the coradical, and since they are not cosemisimple, the coradical is not a sub-Hopf algebra. The coradical can have an arbitrary number of copies on $n \times n$ matrix duals.

Let $H_{p}$ denote the dual Hopf algebra of the following:

2.1. Let $n>1$ be fixed, and assume $k$ contains a primitive $n$th root of unity $\omega$. Let $\mathcal{H}=k\{A, Z\}$ be as in 1.1. Then, for given $p>1$, the ideal $\gamma$ of $\mathcal{H}$ generated by $A^{p n}-1, Z A-\omega A Z$, and $Z^{n}-\left(A^{n}-1\right)$ is a biideal. $A_{p}=\mathcal{H} / \gamma$ is a Hopf algebra with antipode $\Delta$ determined by $\Delta(z)=$ $-z a^{-1}$, and $\left\{a^{i} z^{j}: 0 \leq i<p n, 0 \leq j<n\right\}$ is a basis for $A_{p}$. Thus $\operatorname{dim} H_{p}=$ $\operatorname{dim} A_{p}=p n^{2}$.

One can verify directly the dimension statement in 2.1 by determining the rule for multiplication.

Set $g(X)=X^{p-1}+X^{p-2}+\cdots+1$. Then $X^{p}-1=(X-1) g(X)$. Notice that $b=g\left(a^{n}\right)$ is in the center of $A_{p}$. Furthermore:

2.2. Lemma. Let $\mathbb{M}=(b)$ and $B=A_{p} / M$. Then

(a) $\operatorname{dim} B=(p-1) n^{2}$.

Suppose $p$ is prime to char $k$. Then

(b) $B$ is semisimple, and 
(c) if $k$ is algebraically closed and $\rho \in k$ is a primitive pth root of unity, then $\mathbb{M}_{\rho^{l}}=\left(a^{n}-\rho^{l} 1\right)$ is maximal for $1 \leq l \leq p-1$ and $B \simeq A_{p / M_{\rho}} \times$ $\cdots \times A_{p} / \boldsymbol{m}_{\rho} p-1$.

Proof. (a) Since $a^{n} b=b$ we see that $\left\{b, a b, \ldots, a^{n-1} b\right\}$ spans the set of products of the form $a^{i} b$. The former set is easily seen to be independent, and since $b$ is in the center of $A_{p}$, we see $\operatorname{dim} \mathbb{M}=n^{2}$. Thus $\operatorname{dim} B=$ $\operatorname{codim} M=(p-1) n^{2}$.

(b) Let $\bar{k}$ be an algebraic closure of $k$. If $B \otimes \bar{k}$ is semisimple, then $B$ must be also, so (b) will follow from (c).

(c) If $k$ is algebraically closed then $g(X)=(X-\rho) \cdots\left(X-\rho^{p-1}\right)$. Now $g\left(a^{n}\right) \equiv 0$. Now for each $l$ we have that $a^{n}-\rho^{l} 1$ is in the center of $A_{p}$. Thus $\mathbb{M}=\bigcap \Re_{\rho} l$ and $B \simeq A_{p} / \pi_{\rho} \times \ldots \times A_{p / \pi_{\rho}-1}$ follow easily. It re。 mains to show that $A_{p / m_{\rho} l}=B_{l}$ is simple for $1 \leq l<p$. Notice $\operatorname{dim} B_{l} \leq n^{2}$, so necessarily $\operatorname{dim} B_{l}=n^{2}$ by (a). Thus $B_{l} \simeq M(n, k)$ by 1.3(a). Q.E.D.

Assume $k$ is algebraically closed and that $p$ is prime to char $k$. If $\eta^{p}=1$ set $C_{\eta}=\pi_{\eta}^{\perp}=\left(a^{n}-\eta 1\right)^{\perp}$. Then $C_{\eta}$ is a simple subcoalgebra of $H_{p}$ (in fact $C_{\eta}^{*} \simeq A_{p} / \boldsymbol{m}_{\eta}$ is $n \times n$ matrices) if $\eta \neq 1 ; C_{1}$ is the Hopf alge。 bra of 1.1 since $C_{1}^{*} \simeq A_{p /\left(a^{n}-1\right)}=T_{n}$ and the Hopf algebra $T_{n}$ is self-dual. The following proposition sheds light on the structure of $H_{p}$.

2.3. Proposition. Suppose $k$ is an algebraically closed field which con. tains a primitive nth root of unity, and let $H_{p}$ be as above. Assume that $p$ is prime to char $k$, and let $\rho \in k$ be a primitive pth root of unity. Then

(a) $H_{p}=C_{1} \oplus C_{\rho} \oplus \ldots \oplus C_{\rho p-1}$,

(b) $C_{\mu} C_{\eta} \subseteq C_{\mu \eta}$ for all pth roots of unity $\mu, \eta$. Let $s$ be the antipode of $H_{p}$. Then

(c) $s$ has order $2 n$;

(d) $s\left(C_{\eta}\right) \subseteq C_{\eta-1}$, and $s$ has order $2 n$ on $C_{\rho}$.

Proof. (a) Since $\bigcap_{l=0}^{p-1} \pi_{\rho l}=\left(b\left(a^{n}-1\right)\right)=\left(a^{p n}-1\right)=(0)$, we have (a) by duality.

(b) $\Delta\left(a^{n}-\mu \eta 1\right)=\left(a^{n}-\mu 1\right) \otimes a^{n}+\mu 1 \otimes\left(a^{n}-\eta 1\right)$, so $\Delta\left(a^{n}-\mu \eta 1\right) \subseteq$ $\mathbb{M}_{\mu} \otimes A_{p}+A_{p} \otimes \mathbb{M}_{\eta}$. Since $\Delta$ is an algebra map we conclude that

Thus

$$
\Delta \pi_{\mu \eta} \subseteq \mathbb{M}_{\mu} \otimes A_{p}+A_{p} \otimes \mathbb{M}_{\eta} \text { or } \mathbb{M}_{\mu \eta} \subseteq \mathbb{M}_{\mu} \wedge \mathbb{M}_{\eta}
$$

$$
\left(C_{\mu} C_{\eta}\right)^{\perp}=\mathbb{M}_{\mu} \wedge \mathbb{M}_{\eta} \supseteq \mathbb{M}_{\mu \eta}=C_{\mu \eta}^{\perp}
$$

which proves $C_{\mu} C_{\eta} \subseteq C_{\mu \eta}$.

(c) is clear.

(d) Let $\Delta$ be the antipode of $A_{p}$. Then $\Delta\left(a^{n}-\eta 1\right)=a^{-n}-\eta 1=$ 
$-\eta a^{-n}\left(a^{n}-\eta^{-1} 1\right)$. Thus $\Delta\left(\mathbb{M}_{\eta}\right) \subseteq \mathbb{M}_{\eta}$. . This means $s\left(C_{\eta}\right)=\Delta\left(\mathbb{M}_{\eta}^{\perp}\right) \subseteq$ $\pi_{\eta}^{\perp}-1=C_{\eta}-1$. By (b) $C_{\rho}$ is easily seen to generate $H_{p}$ as an algebra, so that the remainder of (d) follows. Q.E.D.

One can easily show that $\operatorname{Rad} A_{p}=(b z)$ if $p$ is prime to char $k$. If $k=\mathbf{Q}$ or $\mathbf{R}$ and $n=2$, then the dual algebra of the simple subcoalgebra of $H_{2}$ of dimension $>1$ is a division ring.

If char $k=p$ then $\operatorname{Rad} A_{p}=(z)$ and $H_{p}$ is pointed as is $A_{p}$. The degrees of nilpotency of $\operatorname{Rad} A_{p}$ and $\operatorname{Rad} H_{p}$ are different in this case.

3. The invariance of simple subcoalgebras under $t=s^{2}$. We will show that for given $n \geq 5$ there exist nonpointed finite-dimensional Hopf algebras with antipode $s$ of order $2 n$ such that if $C$ is a simple subcoalgebra of dimension $>1$, then $t^{m}(C)=C$ implies $n \mid m$. This is in sharp contrast to [2, 3.3]. Again the Hopf algebras we construct will be generated as algebras by the coradical. The construction is more involved than that of $\$ 2$, but the details will follow a similar pattern. The basic example will be the dual Hopf algebra of 3.1 .

Let $\mathcal{H}=k\left\{A_{0} X, Y\right\}$ be the free $k$-algebra on noncommuting indeterminants $A, X$, and $Y$. Then for $q \geq 1$ fixed, $\mathcal{H}$ has a unique bialgebra structure $(\mathfrak{H}, \Delta, \epsilon)$ where $\Delta: \mathcal{H} \rightarrow \mathcal{H} \otimes \mathcal{H}$ and $\epsilon: \mathcal{H} \rightarrow k$ are determined by

$$
\begin{aligned}
& \triangle A=A \otimes A, \\
& \begin{array}{l}
\Delta X=X \otimes A+1 \otimes X, \quad \text { and } \quad \begin{array}{l}
\epsilon(A)=1, \\
\triangle Y=Y \otimes A^{q}+1 \otimes Y,
\end{array} \quad \epsilon(X)=0=\epsilon(Y) .
\end{array}
\end{aligned}
$$

3.1. Let $n>1$ be fixed and assume that $k$ contains a primitive $n$th root of unity $\omega$. Suppose $q>2$ and $(q, n)=1$. Then the ideal $\gamma$ of $\mathcal{H}$, as above generated by $(p=q-1), A^{p n}-1, X^{n}-\left(A^{n}-1\right), Y^{n}-\left(A^{n}-1\right), X A-\omega A X$, $Y A-\omega^{-q} A Y$, and $X Y-\omega^{q} Y X$, is a bi-ideal, and $A_{q}=\mathcal{H} / \gamma$ is a Hopf algebra with basis $\left\{a^{i} x^{j} y^{l}: 0 \leq i<p n, 0 \leq j, l<n\right\}$. Thus $\operatorname{dim} A_{q}=p n^{3}$.

To see that $\gamma$ is a bi-ideal is straightforward except for possibly showing that $\Delta Y^{n}-\left(Y^{n} \otimes A^{n}+1 \otimes Y^{n}\right) \in \mathcal{H} \otimes \gamma+\gamma \otimes \mathcal{H}$. Now $Y A^{q} \equiv$ $\omega^{-q^{2}} A^{q} Y$ and $\omega^{-q^{2}}$ is a primitive $n$th root of unity since $(q, n)=1$. Thus by $1.2(\mathrm{~b})$,

$$
\Delta Y^{n}-\left(Y^{n} \otimes A^{q n}+1 \otimes Y^{n}\right) \epsilon H \otimes \gamma+\gamma \otimes \mathcal{H} .
$$

But $A^{q n}=A^{n} A^{n(q-1)} \equiv A^{n}$ by assumption. As before the dimension state. ment is straightforward.

Set $g(X)=X^{p-1}+\ldots+1$ and $b=g\left(a^{n}\right)$ as in $\$ 2$. Then we make the following conclusions about $A_{q}$ :

3.2. Lemma. Let $\Re=(b)$ and $B=A_{q} / \Re$ where $p=q-1>1$. Then (a) $\operatorname{dim} B=(p-1) n^{3}$. 
Suppose $p$ is prime to chark. Then

(b) $B$ is semisimple.

Assume further that $k$ is algebraically closed and $\rho \in k$ is a primitive pth root of unity. If $\pi_{\rho l}=\left(a^{n}-\rho^{l} 1\right)$ and $B_{\rho} l=A_{q} \wedge_{\rho} l$, then

$$
B \simeq B_{\rho} \times \cdots \times B_{\rho p-1} \text { and } B_{\rho l} \simeq \underbrace{M(n, k) \times \cdots \times M(n, k)}_{n \circ \text { times }}
$$

for $1 \leq l<p$.

Proof. (a) It is clear by the proof of $2.2\left(\right.$ a) that $\operatorname{dim} \pi=n^{3}$. Thus $\operatorname{dim} B$ $=(p-1) n^{3}$.

(b) will follow from (c) as does 2.2(b) follow from 2.2(c).

(c) $a^{n}-\eta 1$ is in the center of $A_{q}$. So again $\pi=\bigcap_{l=1}^{p-1} \pi_{\rho} l$ and $B \simeq$ $B_{\rho} \times \ldots \times B_{\rho p-1}$. Since $\operatorname{dim} B_{\rho l} \leq n^{3}$, we conclude $\operatorname{dim} B_{\rho l}=n^{3}$ for $1 \leq$ $l<p$. By $1.3(\mathrm{~b}), B_{\rho} l \simeq M(n, k) \times \ldots \times M(n, k)(n$-times $)$ since $\left(\bmod \pi_{\rho l}\right)$ we have $a^{n} \equiv \rho^{l} 1, x^{n} \equiv\left(\rho^{l}-1\right) 1 \equiv y^{n}$, and $\rho^{l}-1 \neq 0$ for $1 \leq l<p$. Q.E.D.

Let $K_{q}=A_{q}^{*}$ where $A_{q}$ is as above.

3.3. Proposition. Let $k$ be an algebraically closed field containing a primitive nth root of unity, where $n>q>2$ and $(q, n)=1$. Let $K_{q}$ be as above. Then:

(a) The antipode $s$ of $K_{q}$ has order $2 n$.

(b) If $p=q-1$ is prime to char $k$, then $K_{q}$ is not pointed.

(c) Suppose $(p, n)=1$ also, and that $C \subseteq K_{q}$ is a simple subcoalgebra. with $\operatorname{dim} C>1$. If $t=s^{2}$ then $t^{m}(C)=C$ implies $n \mid m$.

Proof. (a) Clear by 1.1.

(b) follows by $3.2(\mathrm{c})$ and duality.

(c) Consider $\eta \in \operatorname{Alg}\left(A_{q}, k\right)=G\left(K_{q}\right)$ determined by $\eta(a)=\omega$ and $\eta(x)=$ $0=\eta(y)$. Then it is easy to see that $G\left(K_{q}\right)=(\eta)$ and $\mathfrak{T}=\bigcap_{l=0}^{n-1} \operatorname{ker} \eta^{l}$ is generated as an ideal by $x$ and $y$. If $\pi=(b)$ as in 3.2, then $x^{n} b=0=y^{n} b$. Thus $\Re \mathcal{T}$ is nilpotent. But $\Re+\mathcal{T}=A_{q}$ and $b$ is in the center of $A_{q}$, so $\pi \mathcal{T}=\pi \cap \mathcal{T}$. Therefore $\Re \mathcal{T}=\operatorname{Rad} A_{q}$ by 3.2(b).

Let $C \subseteq K_{q}$ be simple, and $\operatorname{dim} C>1$. Then $C^{\perp}=\mathbb{M}$, where $\mathbb{M}$ is generated by $a^{n}-\rho^{l} 1$ and $y-\xi a^{q} x^{n-q}$, where $\rho$ is a primitive $p$ th root of unity, and $\xi \in k$ satisfies $\left(\xi a^{q} x^{n-q}\right)^{n} \equiv \rho^{l}-1\left(\bmod \Re_{\rho l}\right)$ by $3.2(c)$ and 1.3(b). Let $\Delta$ be the antipode of $A_{q}$. We now determine $\alpha^{2}(\mathcal{K})$. Clearly $\Delta^{2}\left(a^{n}-\rho^{l} 1\right)=a^{n}-\rho^{l} 1$. Now $\Delta(x)=-x a^{-1}$ and $\Delta(y)=-y a^{-q}$, so $\Delta^{2}(x)=$ $a x a^{-1}=\omega^{-1} x$ and $s^{2}(y)=a^{q} y a^{-q}=\omega^{q^{2}} y$. Therefore

$$
\iota^{2}\left(y-\xi a^{q} x^{n-q}\right)=\omega^{q^{2}} y-\xi a^{q}\left(\omega^{-1} x\right)^{n-q}=\omega^{q^{2}}\left(y-\xi \omega^{q-q^{2}} a^{q} x^{n-q}\right) .
$$

If $\lambda=\omega^{q-q^{2}}$, then $\lambda$ is a primitive $n$th root of unity, since $q-q^{2}=-q p$ and $n$ are relatively prime. Let $\mathbb{M}_{\lambda^{k}}$ denote the maximal ideal generated by 
$a^{n}-\rho^{l} 1$ and $y-\xi \lambda^{k} a^{q} x^{n-q}$. What we have shown $\left(\tau=\Delta^{2}\right)$ is that $r\left(M_{\lambda^{k}}\right)=$ $\mathbb{M}_{\lambda^{k+1}}$. Clearly $\mathbb{M}_{\lambda^{k}}=\mathbb{M}_{\lambda^{k^{\prime}}}$ implies $\lambda^{k}=\lambda^{k^{\prime}}$. Since $r^{m}\left(\mathbb{M}_{\lambda^{k}}\right)=\mathbb{M}_{\lambda^{k}}$ if and only if $t^{m}\left(\Re_{\lambda^{k}}^{\perp}\right)=\pi_{\lambda^{k}}^{\perp}$, we have (c). Q.E.D.

The simplest version of 3.3 occurs when $p=2, q=3, n=5$ and $k=\mathbf{Q}$, or char $k>5$.

Remark. It is possible to handle the case of char $k=p=n$ in $\oint_{1}$ using the basic Hopf algebra $A$ of dimension $p^{2}$ described as follows: $A$ is generated by $a, z$ as an algebra subject to $a^{p}=1, z^{p}-z=0,[a, z]=a^{2}-a$; the coalgebra structure is determined by $\Delta a=a \otimes a$ and $\Delta z=z \otimes a+1 \otimes z$. The details are much more complicated and are best handled using the ideas of [6], but the main ideas parallel those of this paper. In particular it is possible to have finite-dimensional Hopf algebras over a field of characteristic $p$ with $r-1$ simple subcoalgebras of dimension $p^{2},(r, p)=1$.

\section{REFERENCES}

1. S. Lang, Algebra, Addison-Wesley, Reading, Mass., 1965. MR 33 \#5416.

2. R. G. Larson, Characters for Hopf algebras, J. Algebra 17 (1971), 352-368. MR 44 \#287。

3. D. E. Radford, The order of the antipode of a finite dimensional Hopf algebra is finite, Amer. J. Math. (to appear).

4. M. E. Sweedler, Hopf algebras, Math. Lecture Note Ser., Benjamin, New York, 1969. MR $40 \# 5705$.

5. E. J. Taft, The order of the antipode of finite-dimensional Hopf algebra, Proc. Nat. Acad. Sci. U.S.A. 68 (1971), 2631-2633. MR 44 \#4075.

6. - Some finite dimensional pointed Hopf algebras with non-semisimple antipode, Proc. Amer. Math. Soc. (to appear). 54911

DEPARTMENT OF MATHEMATICS, LAWRENCE UNIVERSITY, APPLETON, WISCONSIN

Current address: Department of Mathematics, Rutgers University, New Brunswick, New Jersey 08903 